\title{
Ceramides as modulators of cellular and whole-body metabolism
}

\author{
Benjamin T. Bikman and Scott A. Summers
}

Duke-National University of Singapore Graduate Medical School, Singapore.

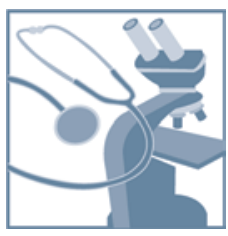

\begin{abstract}
Nearly all stress stimuli (e.g., inflammatory cytokines, glucocorticoids, chemotherapeutics, etc.) induce sphingolipid synthesis, leading to the accumulation of ceramides and ceramide metabolites. While the role of these lipids in the regulation of cell growth and death has been studied extensively, recent studies suggest that a primary consequence of ceramide accumulation is an alteration in metabolism. In both cell-autonomous systems and complex organisms, ceramides modify intracellular signaling pathways to slow anabolism, ensuring that catabolism ensues. These ceramide actions have important implications for diseases associated with obesity, such as diabetes and cardiovascular disease.
\end{abstract}

\section{Introduction}

Obese individuals are at elevated risk of mortality caused by diabetes and cardiovascular disease (1), and a number of mechanisms have been proposed to explain the relationship between increased adiposity and the onset of these deadly pathologies. A hypothesis gaining credibility is that the adipose stores in some obese individuals become saturated and lipotoxic fat metabolites spill over into tissues not suited for lipid storage (2). Of the myriad bioactive lipids that can accumulate in these tissues, the sphingolipid ceramide and its glucosylated metabolites warrant particular attention. Studies conducted in rodent models of diabetes, cardiomyopathy, insulin resistance, atherosclerosis, and hepatic steatosis reveal that inhibiting production of these sphingolipids delays or prevents disease onset (2-4). Thus, abundant evidence suggests that ceramide is one of the most toxic lipids that can accumulate in the obese.

While ceramide has long been known to induce necrosis or apoptosis in numerous types of cells, recent studies indicate that ceramide and its metabolites have profound effects on cellular metabolism (5). In this review, we intend to explore ceramide in this capacity - as a regulator of energy production and nutrient utilization. The metabolic impairments caused by ceramide likely contribute to the tissue dysfunction underlying the numerous diseases associated with obesity.

\section{Ceramide production and degradation}

Ceramides are a family of lipids that consist of sphingosine covalently linked to a fatty acid. Though ceramides were previously believed to be merely structural components of the cell membrane, discoveries over the last few decades reveal that virtually all stress stimuli (e.g., inflammatory mediators, heat, UV radiation, hypoxia, chemotherapeutics, and oxidative stress) increase ceramide production as part of an evolutionarily conserved cellular response (6-10). Three major pathways - de novo synthesis, sphingomyelin hydrolysis, and the salvage pathway - account for the production of ceramide within the cell (Figure 1).

De novo ceramide synthesis. The best characterized of the three pathways is the de novo synthesis of ceramide, which begins with the transfer of a serine residue onto a fatty acyl-CoA via serine pal-

Conflict of interest: The authors have declared that no conflict of interest exists. Citation for this article: J Clin Invest. 2011;121(11):4222-4230. doi:10.1172/JCI57144. mitoyltransferase (SPT) to form 3-keto-sphinganine (3KSn) (11), the 18-carbon backbone on which more complex sphingolipids are built. Three subsequent enzymatic reactions, catalyzed by $3 \mathrm{KSn}$ reductase, (dihydro)ceramide synthases (CerS), and dihydroceramide desaturases (Des1 and -2), convert this transient intermediate into ceramide. Several of the enzymes involved in de novo ceramide synthesis are induced by inflammatory events that are upregulated in obesity (see below). Moreover, pharmacological or genetic inhibition of enzymes required for de novo ceramide synthesis (i.e., SPT, CerS, and Des1) exerts a potent effect on cellular energetics and metabolism. For example, studies conducted using in vitro and in vivo model systems reveal that these manipulations improve postprandial glucose tolerance and reverse insulin resistance caused by glucocorticoids, saturated fatty acids, inflammation, high-fat feeding, and leptin deficiency (12). Since impairment in glucose tolerance places individuals at risk for virtually all the metabolic diseases discussed above, this may be a major mechanism through which ceramide depletion wards off the complications of obesity. A workhorse tool for these studies has been the SPT inhibitor myriocin, a compound originally isolated from an extract of the fruiting bodies of the fungus Isaria sinclairii (辛克莱棒束孢) and its parasitic host larva. I. sinclairii extracts are commonly used in traditional Chinese medicine as nostrums to treat a plethora of conditions, including diabetes, due to their perceived ability to confer eternal youth. Myriocin protects against diabetes in Zucker diabetic fatty (ZDF) rats (12), atherosclerosis in apoE-deficient mice (13), cardiomyopathy in mice with cardiac-specific overexpression of glycosylphosphatidylinositol-anchored (GPI-anchored) human lipoprotein lipase (14), and hepatic steatosis in leptin-deficient (ob/ ob) and diet-induced obese (DIO) mice (15).

We have previously attributed the improved insulin sensitivity and glucose homeostasis caused by myriocin to an increase in insulin signaling, due to ceramide's potent inhibitory effect on the insulin-signaling intermediate Akt (discussed below and ref. 13). However, two recent studies suggest that myriocin may additionally affect fatty acid oxidation. This would predictably lead to the decreased delivery of fat to peripheral tissues and a resulting improvement in glucose utilization. Yang et al. (14) treated three rodent models of obesity $(d b / d b$ and high-fat-fed mice treated at either the onset or after several weeks of high-fat feeding) with myriocin. After 8 weeks of treatment, they found that the inhibition of ceramide synthesis was associated with reduced weight 


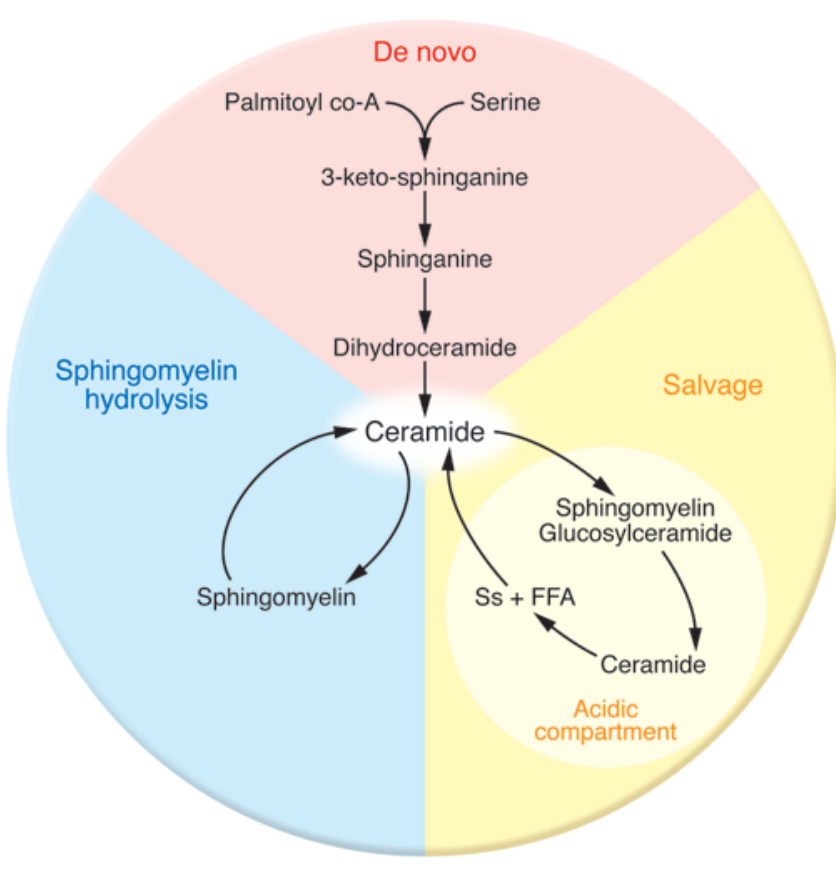

Figure 1

Ceramide can be produced by three pathways. De novo ceramide synthesis depends on the availability of palmitate and serine, while sphingomyelin hydrolysis and ceramide salvage both require an initial supply of ceramide. Ss, sphingosine.

gain compared with nontreated controls. They observed no difference in consumption patterns, but rather found that the discrepancy in weight was associated with a several-fold elevation in levels of adipose uncoupling protein-3 (UCP-3), a mitochondrial protein that increases metabolic rate. Of note, other studies have reported minimal effects of myriocin on weight $(15,16)$, and the reason for the discrepancy is unclear. Ussher et al. (17) found that myriocin reversed a variety of obesity-induced decrements. For example, myriocin-treated mice experienced an increase in oxygen consumption rates, fatty acid oxidation, and heat production when compared with vehicle-treated controls. Interestingly, fenretinide, an inhibitor of dihydroceramide desaturase (18), has also been shown to prevent and reverse diet-induced obesity (19). In particular, in exploring the insulin-sensitizing nature of fenretinide treatment, Preitner et al. (19) found that regardless of when fenretinide supplementation was introduced in the life of DIO mice (i.e., at the onset or after 22 weeks of high-fat feeding), it reduced adiposity, particularly in subcutaneous and visceral fat depots. Despite some discrepancies, these studies suggest that disruption of de novo ceramide synthesis may have a beneficial impact on metabolism.

Sphingomyelin bydrolysis. The second ceramide-generating pathway involves the hydrolysis of the phosphocholine head group from sphingomyelin by a small family of sphingomyelinase (SMase) enzymes distinguished by their $\mathrm{pH}$ optima and/or subcellular location $(20,21)$. As sphingomyelin is the most abundant sphingolipid in mammals, its capacity as a source of ceramide is enormous.

Several noxious stimuli activate SMase, including pathogens, oxidative stress, and inflammatory signals $(2,22)$; the resultant ceramide accumulation is thought to mediate stress-induced apoptosis in a variety of cell types $(23,24)$. Two proinflammatory cytokines, TNF- $\alpha$ and IL-1 $\beta$, activate SMase and induce ceramide accrual $(25,26)$. TNF- $\alpha$ has been widely shown to induce and correlate with insulin resistance (27-30). However, the role of TNF- $\alpha$ in insulin resistance is somewhat controversial, and the importance of ceramide as a mediator of TNF- $\alpha$ action is under debate (31-34).

A limited number of studies have explored the role of SMase in metabolic function and obesity. Samad et al. (35) found that neutral and acid SMases were elevated in adipose of obese rodents, possibly through a TNF- $\alpha$-regulated mechanism. Interestingly, humans with type I Niemann-Pick syndrome, a condition resulting from the absence of acid SMase activity, have trouble gaining and maintaining body weight (36). Mouse models of the condition display weight loss and severe hepatomegaly and hepatosteatosis (37), suggesting inappropriate lipid handling. Deevska et al. (38) explored the ability of acid SMase to alter metabolic function in mice lacking LDL receptors (Ldlr). When challenged with high-fat diet, $L d l r$-KO mice exhibit more severe obesity and dyslipidemia than wild-type mice (39). However, crossing them with mice lacking acid SMase improved glucose and insulin tolerance as well as insulin-stimulated glucose uptake. Interestingly, the doubleknockout mice were somewhat resistant to diet-induced obesity and gained less weight than the mice lacking only $L d l r$.

Salvage pathway. Ceramide can also be produced by the catabolism of other complex sphingolipids, albeit indirectly. Through a series of events referred to as sphingolipid recycling or the salvage pathway, higher-order sphingolipids (sphingomyelin and glycosphingolipids) are degraded within acidic cellular compartments by resident enzymes (acidic SMase and acid $\beta$-glucosidase 1 , respectively) to form ceramide (40-42), which is itself degraded into sphingosine and FFAs that are able to enter the cytosol (43). Once in the cytosol, sphingosine is converted back to ceramide via ceramidase. Though less well known, the salvage pathway may account for over half of the sphingolipid pool within the cell (44). The relative importance of this pathway in obesity-induced ceramide accrual remains unknown. However, overexpression of acid ceramidase (AC) in cultured cells is potently insulin sensitizing, suggesting that lysosomal metabolism of ceramide has beneficial effects (45).

\section{Regulation of ceramide synthesis in obesity}

For some time, ceramide production was thought to be controlled mainly by the availability of its two initial substrates, palmitate and serine. Since these nutrients are likely to be delivered in excess supply in individuals that overeat, the increased substrate availability would be predicted to induce ceramide synthesis. However, recent studies reveal that tissue ceramide levels are regulated by hormonal signals that modulate rates of synthesis and degradation. Inflammatory agonists, which are upregulated in obesity and contribute to insulin resistance and other complications of obesity, are important drivers of ceramide production (31). Conversely, the insulin-sensitizing, antidiabetic, and cardioprotective hormone adiponectin exerts its broad spectrum of actions by degrading ceramide via ceramidase activation (Figure 2 and ref. 46).

Inflammation and ceramide synthesis. All multicellular organisms possess an innate immune system that protects them from invading pathogens. TLRs are essential mediators of this system and respond to distinct molecules found on microbial pathogens. LPS (endotoxin), present on Gram-negative bacteria, is a particularly 
A

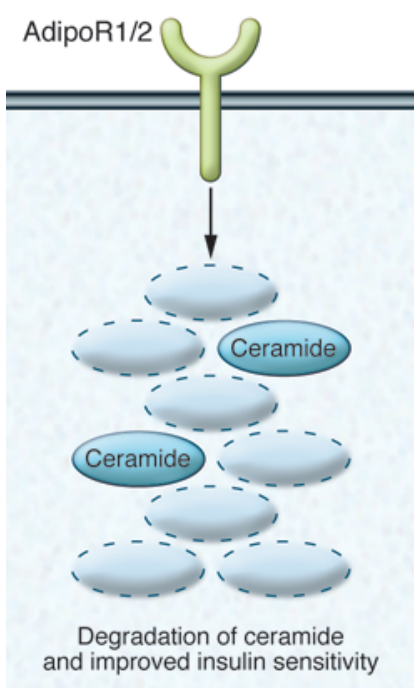

B

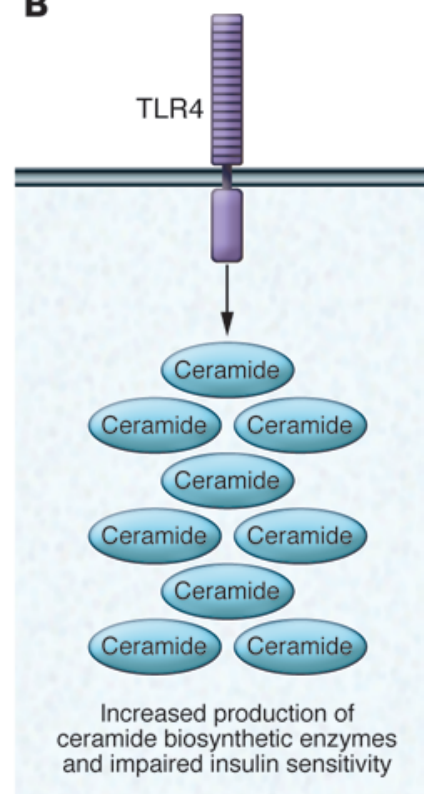

\section{Figure 2}

Ceramide biosynthesis and degradation require specific signals and impart disparate effects. (A) Activation of adiponectin receptors 1 and 2 (AdipoR 1/2) induces synthesis of sphingosine and S1P, which induces an improved metabolic state, characterized by improved insulin sensitivity, reduced inflammation, and AMPK activation. (B) In contrast, activation of TLR4 initiates an inflammatory cascade essential to ceramide biosynthesis and metabolic impingement.

well-characterized activator of TLR4, which initiates phagocytosis of invading organisms and promotes the synthesis and secretion of inflammatory cytokines to recruit additional immune cells to the area. A large number of groups have reported that saturated, but not unsaturated, fatty acids activate TLR4 response (47-52), and Shi et al. (47) demonstrated that this is essential for acute lipid-induced insulin resistance. Subsequent studies in animals fed high-fat diets revealed that TLR4 depletion improves insulin sensitivity in various tissues $(48,50,52,53)$, though the mechanism or mechanisms underlying the connection among fatty acids, TLR4, and insulin action remained unclear until recently.

TLR4 agonists have long been known to promote ceramide synthesis. Through the LIPID MAPS consortium (http://www. lipidmaps.org/), the Merrill group conducted a broad lipidomic profile of cells treated with LPS and demonstrated a selective and robust effect on sphingolipid levels within the cell (54). We have recently shown that mice lacking a functional TLR4 fail to accumulate ceramide in response to elevated saturated fatty acids in insulin-sensitive muscle, liver, and hypothalamus (31). Thus, even when tissues were in a profoundly hyperlipidemic environment, a functioning inflammatory response was necessary to drive ceramide synthesis. More importantly, inhibition of sphingolipid synthesis negated the effects of inflammatory agonists on insulin action (34). Thus, TLR4 was requisite for palmitate-induced insulin resistance and ceramide synthesis, while ceramide synthesis was requisite for TLR4-induced insulin resistance. These data place palmitate, TLR4, and ceramide on a linear pathway that modulates insulin sensitivity.

We have recently shown that LPS and saturated fatty acids, via TLR4, increase levels of transcripts encoding virtually all of the enzymes involved in de novo ceramide synthesis, including SPT subunits, CerS isoforms, and Des1 (31). This transcriptional regula-

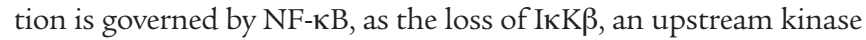
involved in NF-кB activation, blocks the response. Accordingly, treatment of DIO mice with the IKK inhibitor sodium salicylate selectively reduced tissue ceramides and restored insulin sensitivity (31).

Insulin, in addition to having peripheral effects in skeletal muscle and the liver, has actions within the central nervous system. In the arcuate nucleus of the hypothalamus, insulin signaling promotes satiety and reduces hepatic glucose output (55), preventing obesity and maintaining glucose homeostasis (56). The same stimuli that activate TLR4 have been shown to induce hypothalamic insulin resistance $(57,58)$, and we recently identified ceramide as a likely candidate in mediating lipid- and inflammation-induced insulin resistance in this region (31).

Adiponectin and ceramide degradation. The beneficial metabolic effects of the adipocyte-derived hormone adiponectin were previously thought to be mediated by $\operatorname{AMPK}(59,60)$, a serine/threonine kinase that promotes glucose uptake and lipid oxidation. However, the AMPK-centric theory of adiponectin action is challenged by recent findings revealing that liver kinase B1 (LKB1), which is required for AMPK activation, is dispensable for the hormone's effects $(46,61)$. Instead, the Scherer group demonstrated that adiponectin receptors activate cellular ceramidase activity (46), resulting in the degradation of ceramide to form sphingosine. The authors presented data suggesting that this depletion in ceramide could account for a broad spectrum of adiponectin actions, including its ability to prevent apoptosis of pancreatic $\beta$ cells and cardiomyocytes.

We previously demonstrated that overexpression of an AC isoform is insulin sensitizing in vitro (45). An intriguing possibility is that increases in sphingosine by the ceramidase activity of adiponectin receptors lead to elevations in a downstream product, sphingosine 1-phosphate (S1P), which opposes ceramide action. Thus, ceramidase activation would not merely serve to reduce ceramide levels, but also to convert the sphingolipid to a degradation product with antagonistic effects.

$\mathrm{S} 1 \mathrm{P}$ is a bioactive lipid that signals through a family of $\mathrm{G}$ protein-coupled receptors in addition to having other intracellular actions. The opposing action of these two sphingolipids on cell survival prompted researchers to speculate that a cellular S1P-ceramide rheostat is a key regulator of cellular health (62). Recent studies suggest that such a rheostat may also exist for metabolic regulation. In stark contrast to ceramide, S1P promotes nutrient uptake (63) and increases basal and insulin-stimulated glucose uptake $(64,65)$. The molecule has been shown to activate Akt/PKB in a wide variety of cell types $(45,66,67)$. Rapizzi et al. (65) also implicated S1P in insulin receptor activation. Intriguingly, S1P has been shown to activate AMPK (68), and the aforementioned study by the Scherer group suggests that S1P may be necessary for adiponectin-induced AMPK activation. Using a ceramidase inhibitor, the Scherer group (46) found that loss of ceramidase activity in cultured cells prevented AMPK phosphorylation by full-length and truncated globular adiponectin, but did not prevent S1P-induced activation.

\section{Ceramides and nutrient handling}

Recent attention has been placed on ceramide's impact on cellular metabolism as a mechanism driving its action on cell survival. In particular, ceramide was recently shown to kill cells by 


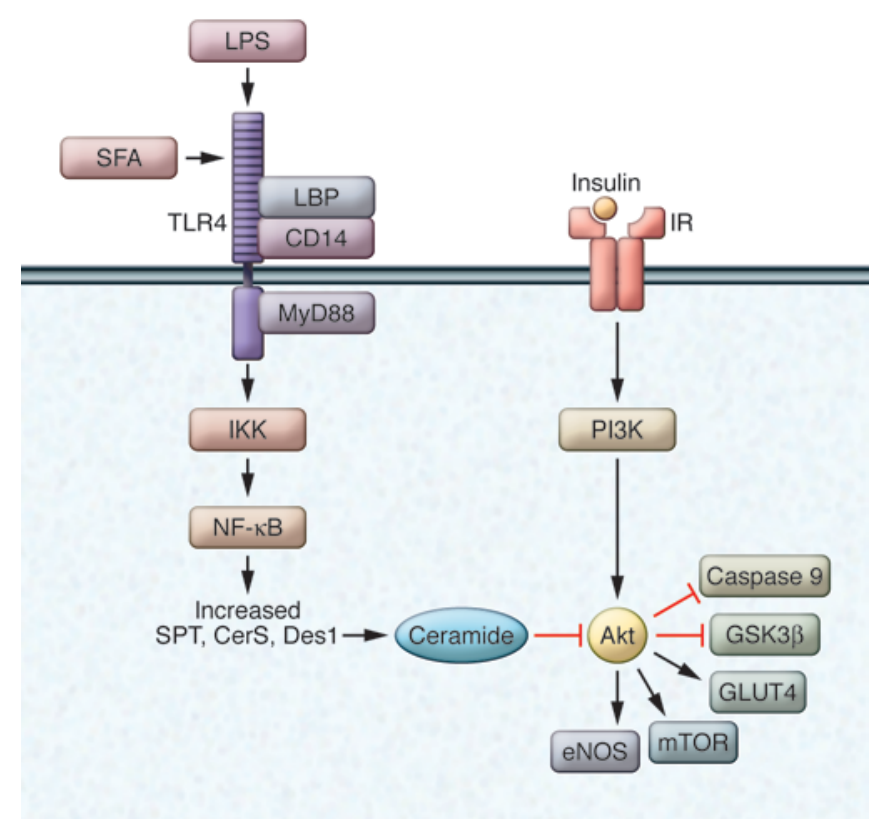

Figure 3

Ceramide accumulation inhibits Akt activation. Central to ceramide's antianabolic effects is its potent inhibition of Akt, resulting in reduced nutrient uptake and progrowth signaling. SFA, saturated fatty acid; LBP, LPS-binding protein.

starving them of nutrients, at least partially by downregulating nutrient transport proteins. Guenther et al. (69) treated cells with methyl pyruvate, a membrane-permeant molecule that can be taken into cells independently of nutrient transporters and oxidized to produce ATP, and this alternative fuel source protected cells from ceramide-induced cell death. The authors speculated that ceramide-dependent starvation is an evolutionarily conserved, cell-autonomous mechanism accounting for the broad spectrum of ceramide actions during times of stress. Moreover, the studies suggest that the autophagic or apoptotic responses to ceramide are secondary to impairments in nutrient delivery. Review of the literature reveals that ceramide indiscriminately affects cellular uptake of all three key dietary nutrients in a variety of cell types and organisms.

Ceramide and glucose. Postprandially, the major hormone controlling circulating glucose levels is insulin. Insulin promotes glucose deposition into skeletal muscle and adipose tissue while simultaneously inhibiting glucose efflux in the liver. In obesity, these actions are compromised. The effect of ceramide as an antagonist of insulin-stimulated glucose uptake has been demonstrated in a variety of cell culture and rodent models of lipid oversupply and obesity. Short-chain ceramide analogs impair insulin-stimulated glucose uptake by blocking the insulinstimulated translocation of the GLUT4 glucose transporter to the plasma membrane (70). Furthermore, rodents treated with myriocin have significantly reduced hepatic and muscle ceramide levels and are protected from saturated fatty acid- and glucocorticoid-induced reductions in glucose infusion rate during a hyperinsulinemic-euglycemic clamp (12). Further, inhibition of ceramide synthesis reduced hepatic glucose output. As discussed above, mice chronically treated with myriocin while consuming a high-fat diet have improved glucose and insulin tolerance as well as enhanced insulin signaling in muscle and liver $(12,14$, 71). Highlighting the critical role for skeletal muscle, the site of the majority of insulin-stimulated postprandial glucose disposal, inhibiting ceramide synthesis in isolated rodent muscle prevents the otherwise significant reduction in insulin-stimulated glucose uptake in the presence of saturated fatty acids (12). Moreover, several groups have found similar results in cell-autonomous systems (72-75). Interestingly, the effect is specific for saturated fatty acids, as unsaturated fatty acids antagonize insulin action via a ceramide-independent mechanism (12).

Ceramide and fatty acids. Despite requiring intracellular fatty acids for its formation, ceramide nonetheless appears to exert a negative effect on lipid uptake. CD36, also referred to as fatty acid translocase, has been implicated in actively transporting fatty acids across the plasma membrane (76). Luan et al. (77) found that treatment of cells with short-chain ceramide inhibits CD36 cell-surface expression in a dose- and time-dependent manner despite having no effect on CD36 mRNA synthesis. The authors speculated that ceramide exerts an inhibitory and specific effect on CD36 membrane translocation, as treating cells with brefeldin A, which inhibits intracellular protein trafficking, had a similar and redundant effect.

Ceramide and amino acids. Several years ago, Skrzypek et al. (78) noticed that cells requiring amino acids were particularly sensitive to sphingolipid-induced growth arrest. In exploring this, they found that sphingoid long-chain bases prevent transport of several amino acids by blocking specific amino acid transporters in Saccharomyces cerevisiae. More recently, Hundal's group found that ceramide inhibits amino acid transport and protein synthesis in mammalian cells (79). Specifically, they observed that both exogenous and endogenous ceramides (i.e., in cells treated with C- 6 ceramide and palmitate, respectively) attenuate basal and insulin-stimulated amino acid transporter in muscle cells by reducing plasma membrane levels of sodiumcoupled neutral amino acid transporter 2 (SNAT2) while leaving total cellular abundance untouched. The reduced SNAT2 membrane levels resulted in a meaningful reduction in the size of the intracellular amino acid pool, with particular effect on glutamine, glutamate, and aspartate, three of the most abundant amino acids within a muscle cell. Ceramide has also been shown to rapidly reduce surface expression of the amino acid transporter 4F2 heavy chain (69).

\section{Ceramide mechanism of action}

Inbibition of Akt. A major mechanism through which ceramide alters cellular metabolism is its dramatic inhibition of Akt $(70,80)$, a serine/threonine kinase that is an obligate intermediate in the signaling pathway linking insulin and other growth factors to the expression, action, and subcellular distribution of nutrient transporters. In addition to mediating nutrient handling, Akt activates anabolic pathways (e.g., glycogen and protein synthesis) while inhibiting catabolism, leading to a marked upregulation in nutrient storage. Akt also activates prosurvival and inhibits autophagic enzymes, leading to a net increase in cellular growth and stability. Accumulating evidence suggests Akt also has roles in cancer (81-83), cardiovascular disease (84), Alzheimer disease $(85,86)$, and type 2 diabetes (87-89).

Ceramide has been shown to oppose nearly all of these Akt actions (ref. 90 and Figure 3). Both exogenous and endogenous ceramides inhibit Akt phosphorylation and activation without 
blocking any upstream signaling events $(73,80)$. In skeletal muscle and fat, this diminution in Akt leads to a decrease in cell-surface GLUT4 expression and a reduction in the activity of anabolic enzymes. The effects of ceramide can be negated by the overexpression of constitutively active forms of Akt, identifying this molecule as an essential mediator of ceramide effects (80).

Ceramide inhibits Akt action by two distinct mechanisms: first, it promotes Akt dephosphorylation via protein phosphatase $2 \mathrm{~A}$ $(91,92)$, which is reportedly a direct target of the sphingolipid; second, it prevents Akt translocation via another putative target, PKC $\zeta(93,94)$. The relative importance of either pathway varies by cell type and is influenced by the amount of caveolae in the cell, as the $\mathrm{PKC} \zeta$-dependent pathway requires functional caveolae (95). Ceramide also inhibits insulin-independent signaling (i.e., IGF-1, PDGF, etc.) to Akt (96). Interestingly, the antagonistic relationship between ceramide and Akt may be reciprocal. Goswami et al. (97) found that cells expressing a constitutively active Akt (myristoylated Akt) failed to accumulate ceramide in response to proapoptotic stimuli.

In addition to regulating GLUT4, Akt modulates the expression or action of other nutrient transport proteins (98-101), prevents a reduction in the ubiquitous glucose transporter GLUT1 when cells are deprived of growth factors, maintains cell-surface levels of the amino acid transporter 4F2hc and amino acid uptake in the absence of growth factors, and preserves cholesterol influx during growth factor withdrawal by sustaining Ldlr expression and membrane localization (100). Additionally, the Bonen lab found a requisite role for Akt in insulin-stimulated increases in fatty acid translocator (CD36) transcript and protein levels (54, 99). Finally, Akt inactivates a number of proapoptotic enzymes, promoting cellular survival. Interestingly, although Akt was not implicated directly, Guenther et al. (69) found that ceramide's inhibitory effect on nutrient transporters was prevented with okadaic acid, a protein phosphatase $2 \mathrm{~A}$ inhibitor, suggesting the involvement of Akt in this effect.

Altered mitochondrial function. Ceramide, in addition to regulating Akt, may modulate mitochondrial function. Due to frequent contamination with an ER-related subcompartment (where ceramide biosynthesis is known to occur), attempts at subcellular fractionation and isolation of mitochondria to determine the presence of ceramide or ceramide-synthesizing enzymes should be carefully evaluated. However, several groups have reported that mitochondria contain a variety of sphingolipids, including ceramide. While ceramides synthesized in the endoplasmic reticulum can be directly transported to other organelles (102), mitochondria themselves carry the enzymatic machinery necessary for ceramide synthesis $(103,104)$. Ceramide is known to interact with mitochondria in inducing apoptotic pathways via increased membrane permeability (for review, see ref. 105).

The presence and synthesis of ceramides in the mitochondria may have particular relevance given ceramide's role in altering components and dynamics of mitochondrial bioenergetics. Di Paola et al. (106) found that the ceramide-induced perturbation of mitochondrial membrane structure elicits a reduction in state 4 respiration and inhibition of electron transport complex I. Others report a reduction in complex III activity in the presence of elevated ceramides, but not dihydroceramides (107). Not surprisingly, the disruption of electron transport by ceramide results in elevated reactive oxygen species (108), which can have a wide range of deleterious consequences, including the induction of insulin resistance (109). In vivo models support the conclusion that inhibition of ceramide improves mitochondrial function (71).

Interestingly, JNK, a known metabolic irritant and initiator of inflammation-induced insulin resistance (110), has been shown to induce expression of ceramide biosynthetic enzymes in the mitochondria. Moreover, inhibition of JNK results in diminished ceramide levels and restoration of mitochondrial electron transport function (111).

\section{Glucosylated ceramides in the regulation of metabolism}

Some time ago, we demonstrated that an inhibitor of ceramide glucosylation antagonized insulin-stimulated Akt activation while inducing ceramide accumulation in cultured myotubes. These data suggested that ceramide, and not a glucosylated ceramide metabolite, was the primary mediator of insulin resistance (73). However, recent studies conducted in vivo using newer generation glucosylceramide synthesis inhibitors that do not induce ceramide suggest a different conclusion. Two different glucosylceramide synthase (GCS) inhibitors, when given to rodents, have an array of beneficial effects on metabolism. Aerts et al. found that a GCS inhibitor improved hepatic insulin sensitivity (112) and prolonged treatment reduced hepatic inflammation with diet-induced obesity (113); similar improvements have been observed with a second GCS inhibitor (114). These compounds appear to reverse many of the complications associated with obesity, including diabetes (112), adipose inflammation (113), hepatic steatosis (115), and atherosclerosis (114), though reports on the latter are controversial (116). GM3 ganglioside, a higher order glycosphingolipid, may be the primary antagonist of insulin action. Tagami et al. (117) found that inhibition of GM3 synthesis reversed TNF- $\alpha$-induced insulin resistance in cultured 3T3-L1 adipocytes, while the addition of GM3 analogs impaired insulin action.

Of the various sphingolipid disorders evident in humans, the lack of glucosylceramidase is the most common (118). Known as Gaucher disease, it is a disorder defined by the inability to catabolize glucosylceramides, leading to glucosylceramide accumulation in tissue and in circulation (119). The inability to degrade glucosylceramide into its constituent parts, ceramide and glucose, yields a proinflammatory and insulin-resistant phenotype (120). The condition has a particularly vicious impact on the liver, resulting in marked hepatosplenomegaly and severe hepatic insulin resistance (121). Interestingly, and possibly exacerbating these symptoms, Gaucher patients present lower circulating adiponectin levels (122). Similarly to ceramide, glucosylceramide appears to also have effects unrelated to its impact on insulin. After observing and noting increased glucosylceramides in adipose of mammals and drosophila, Kohyama-Koganeya et al. (123) questioned the role of glucosylceramides in regulating energy storage. They found that GCS overexpression increased lipid and carbohydrate storage in the drosophila fat body, essentially giving the flies a "pot belly." In contrast, glucosylceramide accumulation in Gaucher patients has no effect on obesity prevalence, and patients tend to weigh less than the general population (118). However, restoration of glucosylceramidase action via intravenous infusion of a recombinant glucosylceramidase not only eliminated the weight discrepancy (i.e., patients gained weight), but also increased risk of type 2 diabetes and diminished peripheral insulin sensitivity 
(118), returning the patient to a more classic obesity-induced insulin-resistant condition. Thus, in an effort to improve health by restoring the ability of the cell to degrade glucosylceramide into ceramide, the patient trades one set of metabolic complications for another.

\section{Ceramides and the $\beta$ cell}

Thus far, we have mainly considered the roles for ceramides and glucosylceramides as cell-autonomous modulators of metabolic flux. However, studies in the pancreatic $\beta$ cell reveal that ceramide also modulates insulin synthesis and cellular apoptosis, therefore limiting anabolism by altering the postprandial hormonal milieu.

Ceramide has been shown to accumulate in $\beta$ cells exposed to either saturated fatty acids or to a glucolipotoxic environment (124). One of the earliest reports exploring ceramide's effects on the $\beta$ cell found that exogenous short-chain and sphingomyelinderived ceramides inhibited insulin release and $\beta$ cell proliferation in vitro, though the mechanism was unclear (125). Subsequently, Kelpe et al. (126) found that increasing concentrations of palmitate induced ceramide accrual and reduced preproinsulin mRNA. Through the addition of cell permeable C-2 ceramide and cotreatment of palmitate-treated cells with inhibitors of de novo ceramide synthesis (myriocin, fumonisin B1, and cycloserine), they demonstrated that ceramide reduced preproinsulin transcript levels. Similar results were found by Guo et al. (127), who induced ceramide accumulation in a rat $\beta$ cell line (INS-1) by preventing its metabolism to sphingosine with the ceramidase inhibitor $n$-oleoylethanolamine (NOE). Treating INS- 1 cells with palmitate and NOE induced ceramide accumulation and reduced proinsulin 1 and 2 mRNA.

Though islets do not undergo apoptosis when exposed to exogenous FFAs, those isolated from mice lacking a functional leptin axis undergo ceramide-dependent apoptosis following palmitate treatment. Moreover, studies in ZDF rats reveal that inhibition of ceramide synthesis prevents $\beta$ cell destruction in this spontaneous model of diabetes. The finding was first observed by the Unger group, who demonstrated that the SPT inhibitor cycloserine prevented lipotoxicity in islets isolated from $Z D F$ rats, while preventing pancreatic $\beta$ cell destruction in vivo $(12,128)$. This finding caused the Unger group to describe ceramides as "the most important of the deleterious routes” mediating lipotoxic events (129). Similar findings were obtained by our group using myriocin, a more selective inhibitor of SPT (12). Further, the very recent work by the Scherer lab (46) confirms that INS- $1 \beta$ cells enriched with ceramides have impaired survival. A fascinating aspect of the Scherer study is that ceramide- and palmitate-induced apoptosis is prevented by cotreatment with S1P, suggesting that the ceramide-S1P rheostat is functional in this cell type.

\section{Concluding remarks}

Ceramide accumulation in cells alters a large number of signal transduction events, leading to an increase in cell growth and sur- vival. What has been less appreciated until recently is that this increase in ceramide is accompanied by a marked shift in cellular bioenergetics that serves to inhibit nutrient uptake and anabolic metabolism in a wide array of tissues. This series of events appears to be critically important in the onset and progression of metabolic disease, as the impairment in nutrient utilization exacerbates the pathogenic consequences of obesity. However, a few outstanding questions still remain. (a) Which ceramides and ceramide metabolites play the most quantitatively significant role in metabolic adaptation, and which are most critically important for disease onset? (b) What tissues are most sensitive to ceramide deposition? (c) How are small changes in ceramide and glucosylceramides sensed intracellularly? How do small increases have such profound effects on metabolism?

Of course, the most relevant question today is whether ceramide deposition plays an important role in the pathogenesis of metabolic diseases in humans. Thus far, the only studies conducted in people have been correlative in nature, comparing sphingolipid levels in serum or tissues with one or more disease readouts. In comparison, studies in mice have been able to apply interventional techniques (e.g., ceramide synthesis inhibition) to determine the importance of ceramide in metabolic disease. In the majority of human studies, ceramides in serum, liver, adipose tissue, or muscle have positively associated with parameters associated with metabolic disease (e.g., insulin resistance, hepatic steatosis, etc.) (130-133). However, some studies have shown no such relationship (134). Whether these data mean that ceramide is uniquely relevant to the mouse, and not to people, is open for debate. Alternative explanations are that ceramide metabolites, and not ceramides themselves, are the relevant antagonists of tissue function in the obese or that scientists are simply looking in the wrong tissue or subcellular locale. Addressing the issues listed above could provide clarity in this dilemma.

In summary, inhibition of ceramide synthesis has a remarkably broad spectrum of metabolic benefits in obese rodents and therefore holds promise as a therapeutic strategy for treating a variety of metabolic diseases. The conserved metabolic adaptations caused by ceramide are likely an evolutionarily conserved response to a suboptimal extracellular environment and could be a major mechanism underlying the onset of metabolic disease. But more work must be done to develop an understanding of the nuanced regulatory events linking ceramide deposition to metabolic adaptation as well as to determine the validity of ceramide modulation as a strategy for relieving disease symptoms in the obese.

Address correspondence to: Scott Summers, Duke-NUS Graduate Medical School, 8 College Road, Singapore, UNK 169857. Phone: 65.6516.8793; Fax: 65.6534.8632;E-mail: scott.summers@ duke-nus.edu.sg.

Benjamin T. Bikman's present address is: Brigham Young University, Provo, Utah, USA.

\footnotetext{
1. Flegal KM, Graubard BI, Williamson DF, Gail MH. Cause-specific excess deaths associated with underweight, overweight, and obesity. JAMA. 2007;298(17):2028-2037.

2. Summers SA. Ceramides in insulin resistance and lipotoxicity. Prog Lipid Res. 2006;45(1):42-72.
}

3. Polya JB, Parsons RS. Free ceramide in blood and its relevance to atherosclerosis. I. Med J Aust. 1973;1(18):873-879.

4. Bismuth J, Lin P, Yao Q, Chen C. Ceramide: a common pathway for atherosclerosis? Atherosclerosis. 2008;196(2):497-504.
5. Guenther GG, Edinger AL. A new take on ceramide: starving cells by cutting off the nutrient supply. Cell Cycle. 2009;8(8):1122-1126.

6. Rozenova KA, Deevska GM, Karakashian AA, Nikolova-Karakashian MN. Studies on the role of acid sphingomyelinase and ceramide in the 
regulation of tumor necrosis factor alpha (TNFalpha)-converting enzyme activity and TNFalpha secretion in macrophages. J Biol Chem. 2010; 285(27):21103-21113.

7. Li X, Becker KA, Zhang Y. Ceramide in redox signaling and cardiovascular diseases. Cell Physiol Biochem. 2010;26(1):41-48.

8. Hannun YA, Obeid LM. The Ceramide-centric universe of lipid-mediated cell regulation: stress encounters of the lipid kind. J Biol Chem. 2002;277(29):25847-25850.

9. Sawai H, Hannun YA. Ceramide and sphingomyelinases in the regulation of stress responses. Chem Phys Lipids. 1999;102(1-2):141-147.

10. Hannun YA, Luberto C. Ceramide in the eukaryotic stress response. Trends Cell Biol. 2000;10(2):73-80.

11. Holland WL, Summers SA. Sphingolipids, insulin resistance, and metabolic disease: new insights from in vivo manipulation of sphingolipid metabolism. Endocr Rev. 2008;29(4):381-402.

12. Holland WL, et al. Inhibition of ceramide synthesis ameliorates glucocorticoid-, saturated-fat-, and obesity-induced insulin resistance. Cell Metab. 2007;5(3):167-179.

13. Summers SA, Garza LA, Zhou H, Birnbaum MJ. Regulation of insulin-stimulated glucose transporter GLUT4 translocation and Akt kinase activity by ceramide. Mol Cell Biol. 1998; 18(9):5457-5464.

14. Yang G, Badeanlou L, Bielawski J, Roberts AJ, Hannun YA, Samad F. Central role of ceramide biosynthesis in body weight regulation, energy metabolism, and the metabolic syndrome. Am J Physiol Endocrinol Metab. 2009;297(1):E211-E224.

15. Park TS, Rosebury W, Kindt EK, Kowala MC, Panek RL. Serine palmitoyltransferase inhibitor myriocin induces the regression of atherosclerotic plaques in hyperlipidemic ApoE-deficient mice. Pharmacol Res. 2008;58(1):45-51.

16. Park TS, et al. Inhibition of sphingomyelin synthesis reduces atherogenesis in apolipoprotein E-knockout mice. Circulation. 2004;110(22):3465-3471.

17. Ussher JR, et al. Inhibition of de novo ceramide synthesis reverses diet-induced insulin resistance and enhances whole-body oxygen consumption. Diabetes. 2010;59(10):2453-2464.

18. Zheng W, et al. Ceramides and other bioactive sphingolipid backbones in health and disease: lipidomic analysis, metabolism and roles in membrane structure, dynamics, signaling and autophagy. Biochim Biophys Acta. 2006;1758(12):1864-1884.

19. Preitner F, Mody N, Graham TE, Peroni OD, Kahn BB. Long-term Fenretinide treatment prevents high-fat diet-induced obesity, insulin resistance, and hepatic steatosis. Am J Physiol Endocrinol Metab. 2009;297(6):E1420-E1429.

20. Boulgaropoulos B, Amenitsch H, Laggner P, Pabst G. Implication of sphingomyelin/ceramide molar ratio on the biological activity of sphingomyelinase. Biophys J. 2010;99(2):499-506.

21. Claus RA, Dorer MJ, Bunck AC, Deigner HP. Inhibition of sphingomyelin hydrolysis: targeting the lipid mediator ceramide as a key regulator of cellular fate. Curr Med Chem. 2009;16(16):1978-2000.

22. Zeidan YH, Hannun YA. The acid sphingomyelinase/ceramide pathway: biomedical significance and mechanisms of regulation. Curr Mol Med. 2009;10(5):454-466.

23. Smith EL, Schuchman EH. The unexpected role of acid sphingomyelinase in cell death and the pathophysiology of common diseases. FASEB J. 2008;22(10):3419-3431.

24. Pena LA, Fuks Z, Kolesnick R. Stress-induced apoptosis and the sphingomyelin pathway. Biochem Pharmacol. 1997;53(5):615-621.

25. Dressler KA, Mathias S, Kolesnick RN. Tumor necrosis factor-alpha activates the sphingomyelin signal transduction pathway in a cell-free system.
Science. 1992;255(5052):1715-1718.

26. Wiegmann K, Schutze S, Machleidt T, Witte D, Kronke M. Functional dichotomy of neutral and acidic sphingomyelinases in tumor necrosis factor signaling. Cell. 1994;78(6):1005-1015.

27. Hotamisligil GS, Shargill NS, Spiegelman BM. Adipose expression of tumor necrosis factor-alpha: direct role in obesity-linked insulin resistance. Science. 1993;259(5091):87-91.

28. Hotamisligil GS, Arner P, Caro JF, Atkinson RL, Spiegelman BM. Increased adipose tissue expression of tumor necrosis factor-alpha in human obesity and insulin resistance. J Clin Invest. 1995;95(5):2409-2415.

29. Hofmann C, et al. Altered gene expression for tumor necrosis factor-alpha and its receptors during drug and dietary modulation of insulin resistance. Endocrinology. 1994;134(1):264-270.

30. Stephens JM, Lee J, Pilch PF. Tumor necrosis factor-alpha-induced insulin resistance in 3T3-L1 adipocytes is accompanied by a loss of insulin receptor substrate-1 and GLUT4 expression without a loss of insulin receptor-mediated signal transduction. J Biol Chem. 1997;272(2):971-976.

31. Holland WL, et al. Lipid-induced insulin resistance mediated by the proinflammatory receptor TLR4 requires saturated fatty acid-induced ceramide biosynthesis in mice. J Clin Invest. 2011; 121(5):1858-1870.

32. Fernandez-Veledo S, Hernandez R, Teruel T, Mas JA, Ros M, Lorenzo M. Ceramide mediates TNFalpha-induced insulin resistance on GLUT4 gene expression in brown adipocytes. Arch Physiol Biochem. 2006;112(1):13-22.

33. Teruel T, Hernandez R, Lorenzo M. Ceramide mediates insulin resistance by tumor necrosis factor-alpha in brown adipocytes by maintaining Akt in an inactive dephosphorylated state. Diabetes. 2001;50(11):2563-2571.

34. Grigsby RJ, Dobrowsky RT. Inhibition of ceramide production reverses TNF-induced insulin resistance. Biochem Biophys Res Commun. 2001;287(5):1121-1124.

35. Samad F, Hester KD, Yang G, Hannun YA, Bielawski J. Altered adipose and plasma sphingolipid metabolism in obesity: a potential mechanism for cardiovascular and metabolic risk. Diabetes. 2006;55(9):2579-2587.

36. Crocker AC, Farber S. Niemann-Pick disease: a review of eighteen patients. Medicine (Baltimore). 1958;37(1):1-95.

37. Garver WS, et al. Characterization of liver disease and lipid metabolism in the Niemann-Pick C1 mouse. J Cell Biochem. 2007;101(2):498-516.

38. Deevska GM, et al. Acid Sphingomyelinase Deficiency Prevents Diet-induced Hepatic Triacylglycerol Accumulation and Hyperglycemia in Mice. J Biol Chem. 2009;284(13):8359-8368.

39. Schreyer SA, Vick C, Lystig TC, Mystkowski P, LeBoeuf RC. LDL receptor but not apolipoprotein E deficiency increases diet-induced obesity and diabetes in mice. Am J Physiol Endocrinol Metab. 2002;282(1):E207-E214.

40. Kitatani K, Idkowiak-Baldys J, Hannun YA. The sphingolipid salvage pathway in ceramide metabolism and signaling. Cell Signal. 2008;20(6):1010-1018.

41. Kitatani K, et al. Involvement of acid beta-glucosidase 1 in the salvage pathway of ceramide formation. J Biol Chem. 2009;284(19):12972-12978.

42. Riboni L, Prinetti A, Bassi R, Tettamanti G. Formation of bioactive sphingoid molecules from exogenous sphingomyelin in primary cultures of neurons and astrocytes. FEBS Lett. 1994; 352(3):323-326.

43. Riboni L, Bassi R, Caminiti A, Prinetti A, Viani P, Tettamanti G. Metabolic fate of exogenous sphingosine in neuroblastoma neuro2A cells. Dosedependence and biological effects. Ann NY Acad Sci.
1998;845:46-56.

44. Gillard BK, Clement RG, Marcus DM. Variations among cell lines in the synthesis of sphingolipids in de novo and recycling pathways. Glycobiology. 1998;8(9):885-890.

45. Chavez JA, Holland WL, Bar J, Sandhoff K, Summers SA. Acid ceramidase overexpression prevents the inhibitory effects of saturated fatty acids on insulin signaling. $J$ Biol Chem. 2005; 280(20):20148-20153.

46. Holland WL, et al. Receptor-mediated activation of ceramidase activity initiates the pleiotropic actions of adiponectin. Nat Med. 2010;17(1):55-63.

47. Shi H, Kokoeva MV, Inouye K, Tzameli I, Yin H, Flier JS. TLR4 links innate immunity and fatty acid-induced insulin resistance. J Clin Invest. 2006; 116(11):3015-3025.

48. Kim F, et al. Toll-like receptor-4 mediates vascular inflammation and insulin resistance in diet-induced obesity. Circ Res. 2007;100(11):1589-1596.

49. Reyna SM, et al. Elevated toll-like receptor 4 expression and signaling in muscle from insulin-resistant subjects. Diabetes. 2008;57(10):2595-2602.

50. Tsukumo DM, et al. Loss-of-function mutation in Toll-like receptor 4 prevents diet-induced obesity and insulin resistance. Diabetes. 2007; 56(8):1986-1998.

51. Davis JE, Gabler NK, Walker-Daniels J, Spurlock ME. The c-Jun $\mathrm{N}$-terminal kinase mediates the induction of oxidative stress and insulin resistance by palmitate and toll-like receptor 2 and 4 ligands in 3T3-L1 adipocytes. Horm Metab Res. 2009;41(7):523-530.

52. Davis JE, Gabler NK, Walker-Daniels J, Spurlock ME. Tlr-4 deficiency selectively protects against obesity induced by diets high in saturated fat. Obesity (Silver Spring). 2008;16(6):1248-1255.

53. Poggi $\mathrm{M}$, et al. $\mathrm{C} 3 \mathrm{H} / \mathrm{HeJ}$ mice carrying a toll-like receptor 4 mutation are protected against the development of insulin resistance in white adipose tissue in response to a high-fat diet. Diabetologia. 2007;50(6):1267-1276.

54. Sims K, et al. Kdo2-lipid A, a TLR4-specific agonist, induces de novo sphingolipid biosynthesis in RAW264.7 macrophages, which is essential for induction of autophagy. J Biol Chem. 2010;285(49):38568-38579.

55. Obici S, Zhang BB, Karkanias G, Rossetti L. Hypothalamic insulin signaling is required for inhibition of glucose production. Nature Medicine. 2002;8(12):1376-1382.

56. Bruning JC, et al. Role of brain insulin receptor in control of body weight and reproduction. Science. 2000;289(5487):2122-2125.

57. Posey KA, et al. Hypothalamic proinflammatory lipid accumulation, inflammation, and insulin resistance in rats fed a high-fat diet. Am J Physiol Endocrinol Metab. 2009;296(5):E1003-E1012.

58. Benoit SC, et al. Palmitic acid mediates hypothalamic insulin resistance by altering PKC-theta subcellular localization in rodents. J Clin Invest. 2009;119(9):2577-2589.

59. Yamauchi T, et al. Adiponectin stimulates glucose utilization and fatty-acid oxidation by activating AMP-activated protein kinase. Nat Med. 2002;8(11):1288-1295.

60. Bikman BT, Zheng D, Reed MA, Hickner RC, Houmard JA, Dohm GL. Lipid-induced insulin resistance is prevented in lean and obese myotubes by AICAR treatment. Am J Physiol Regul Integr Comp Physiol. 2010;298(6):R1692-R1699.

61. Miller RA, et al. Adiponectin suppresses gluconeogenic gene expression in mouse hepatocytes independent of LKB1-AMPK signaling. J Clin Invest. 2011;121(6):2518-2528

62. Lavieu $\mathrm{G}$, et al. Is autophagy the key mechanism by which the sphingolipid rheostat controls the cell fate decision? Autophagy. 2007;3(1):45-47. 
63. Yea K, et al. Lysophosphatidic acid regulates blood glucose by stimulating myotube and adipocyte glucose uptake. J Mol Med. 2008;86(2):211-220.

64. Ma MM, et al. Sphingosine kinase 1 participates in insulin signalling and regulates glucose metabolism and homeostasis in KK/Ay diabetic mice. Dia betologia. 2007;50(4):891-900.

65. Rapizzi E, Taddei ML, Fiaschi T, Donati C, Bruni $\mathrm{P}$, Chiarugi P. Sphingosine 1-phosphate increases glucose uptake through trans-activation of insulin receptor. Cell Mol Life Sci. 2009;66(19):3207-3218.

66. Spiegel S, Milstien S. Sphingosine-1-phosphate: an enigmatic signalling lipid. Nat Rev Mol Cell Biol. 2003;4(5):397-407.

67. Morales-Ruiz M, et al. Sphingosine 1-phosphate activates Akt, nitric oxide production, and chemotaxis through a Gi protein/phosphoinositide 3-kinase pathway in endothelial cells. J Biol Chem. 2001;276(22):19672-19677.

68. Levine YC, Li GK, Michel T. Agonist-modulated regulation of AMP-activated protein kinase (AMPK) in endothelial cells. Evidence for an AMPK -> Rac1 -> Akt -> endothelial nitric-oxide synthase pathway. J Biol Chem. 2007;282(28):20351-20364.

69. Guenther GG, Peralta ER, Rosales KR, Wong SY, Siskind LJ, Edinger AL. Ceramide starves cells to death by downregulating nutrient transporter proteins. Proc Natl Acad Sci US A. 2008;105(45):17402-17407.

70. Summers SA, Garza LA, Zhou H, Birnbaum MJ. Regulation of insulin-stimulated glucose transporter GLUT4 translocation and Akt kinase activity by ceramide. Mol Cell Biol. 1998; 18(9):5457-5464.

71. Ussher JR, et al. Inhibition of de novo ceramide synthesis reverses diet-induced insulin resistance and enhances whole body oxygen consumption. Diabetes. 2010;59(10):2453-2464.

72. Chavez JA, Summers SA. Characterizing the effects of saturated fatty acids on insulin signaling and ceramide and diacylglycerol accumulation in 3T3L1 adipocytes and C2C12 myotubes. Arch Biochem Biophys. 2003;419(2):101-109.

73. Chavez JA, et al. A role for ceramide, but not diacylglycerol, in the antagonism of insulin signal transduction by saturated fatty acids. J Biol Chem. 2003;278(12):10297-10303

74. Cazzolli R, Carpenter L, Biden TJ, Schmitz-Peiffer C. A role for protein phosphatase 2A-like activity, but not atypical protein kinase Czeta, in the inhibition of protein kinase B/Akt and glycogen synthesis by palmitate. Diabetes. 2001; 50(10):2210-2218

75. Schmitz-Peiffer C, Craig DL, Biden TJ. Ceramide generation is sufficient to account for the inhibition of the insulin-stimulated PKB pathway in C2C12 skeletal muscle cells pretreated with palmitate. J Biol Chem. 1999;274(34):24202-24210.

76. Schwenk RW, Holloway GP, Luiken JJ, Bonen A, Glatz JF. Fatty acid transport across the cell membrane: regulation by fatty acid transporters. Prostaglandins Leukot Essent Fatty Acids. 2010;82(4-6):149-154

77. Luan Y, Griffiths HR. Ceramides reduce CD36 cell surface expression and oxidised LDL uptake by monocytes and macrophages. Arch Biochem Biophys. 2006;450(1):89-99.

78. Skrzypek MS, Nagiec MM, Lester RL, Dickson RC. Inhibition of amino acid transport by sphingoid long chain bases in Saccharomyces cerevisiae. J Biol Chem. 1998;273(5):2829-2834.

79. Hyde R, Hajduch E, Powell DJ, Taylor PM, Hundal HS. Ceramide down-regulates System A amino acid transport and protein synthesis in rat skeletal muscle cells. FASEB J. 2005;19(3):461-463.

80. Zhou H, Summers SA, Birnbaum MJ, Pittman RN Inhibition of Akt kinase by cell-permeable ceramide and its implications for ceramide-induced apoptosis. J Biol Chem. 1998;273(26):16568-16575.

81. Vivanco I, Sawyers CL. The phosphatidylinositol
3-Kinase AKT pathway in human cancer. Nat Rev Cancer. 2002;2(7):489-501.

82. Generali D, et al. Down-regulation of phosphatidylinositol 3'-kinase/AKT/molecular target of rapamycin metabolic pathway by primary letrozole-based therapy in human breast cancer. Clin Cancer Res. 2008;14(9):2673-2680.

83. Gershtein ES, Scherbakov AM, Shatskaya VA, Kushlinsky NE, Krasilnikov MA. Phosphatidylinositol 3-kinase/AKT signalling pathway components in human breast cancer: clinicopathological correlations. Anticancer Res. 2007; 27(4A):1777-1782

84. Sugden PH, Clerk A. Akt like a woman: gender differences in susceptibility to cardiovascular disease. Circ Res. 2001;88(10):975-977.

85. Griffin RJ, et al. Activation of Akt/PKB, increased phosphorylation of Akt substrates and loss and altered distribution of Akt and PTEN are features of Alzheimer's disease pathology. J Neurochem. 2005;93(1):105-117.

86. Rickle A, Bogdanovic N, Volkman I, Winblad B, Ravid R, Cowburn RF. Akt activity in Alzheimer's disease and other neurodegenerative disorders. Neuroreport. 2004;15(6):955-959.

87. Krook A, Roth RA, Jiang XJ, Zierath JR, WallbergHenriksson H. Insulin-stimulated Akt kinase activity is reduced in skeletal muscle from NIDDM subjects. Diabetes. 1998;47(8):1281-1286.

88. Cho $\mathrm{H}$, et al. Insulin resistance and a diabetes mellitus-like syndrome in mice lacking the protein kinase Akt2 (PKB beta). Science. 2001; 292(5522):1728-1731.

89. Cho H, Thorvaldsen JL, Chu Q, Feng F, Birnbaum MJ. Akt1/PKBalpha is required for normal growth but dispensable for maintenance of glucose homeostasis in mice. J Biol Chem. 2001;276(42):38349-38352.

90. Stoica BA, Movsesyan VA, Lea PMt, Faden AI. Ceramide-induced neuronal apoptosis is associated with dephosphorylation of Akt, BAD, FKHR, GSK-3beta, and induction of the mitochondrialdependent intrinsic caspase pathway. Mol Cell Newrosci. 2003;22(3):365-382.

91. Salinas M, Lopez-Valdaliso R, Martin D, Alvarez A, Cuadrado A. Inhibition of PKB/Akt1 by C2ceramide involves activation of ceramide-activated protein phosphatase in PC12 cells. Mol Cell Neurosci. 2000;15(2):156-169.

92. Dobrowsky RT, Kamibayashi C, Mumby MC, Hannun YA. Ceramide activates heterotrimeric protein phosphatase 2A. J Biol Chem. 1993; 268(21):15523-15530.

93. Stratford S, DeWald DB, Summers SA. Ceramide dissociates 3'-phosphoinositide production from pleckstrin homology domain translocation. Biochem J. 2001;354(pt 2):359-368.

94. Powell DJ, Hajduch E, Kular G, Hundal HS. Ceramide disables 3-phosphoinositide binding to the pleckstrin homology domain of protein kinase $\mathrm{B}$ (PKB)/Akt by a PKCzeta-dependent mechanism. Mol Cell Biol. 2003;23(21):7794-7808.

95. Hajduch E, et al. Targeting of PKCzeta and PKB to caveolin-enriched microdomains represents a crucial step underpinning the disruption in PKB-directed signalling by ceramide. Biochem J. 2008;410(2):369-379

96. Strle K, et al. Proinflammatory cytokine impairment of insulin-like growth factor I-induced protein synthesis in skeletal muscle myoblasts requires ceramide. Endocrinology. 2004; 145(10):4592-4602.

97. Goswami R, Kilkus J, Dawson SA, Dawson G. Overexpression of Akt (protein kinase B) confers protection against apoptosis and prevents formation of ceramide in response to pro-apoptotic stimuli. J Neurosci Res. 1999;57(6):884-893.

98. Klip A. The many ways to regulate glucose transporter 4. Appl Physiol Nutr Metab. 2009;34(3):481-487.
99. Chabowski A, et al. Insulin stimulates fatty acid transport by regulating expression of FAT/CD36 but not FABPpm. Am J Physiol Endocrinol Metab. 2004;287(4):E781-E789.

100.Edinger AL, Thompson CB. Akt maintains cell size and survival by increasing mTOR-dependent nutrient uptake. Mol Biol Cell. 2002;13(7):2276-2288.

101. Hyde R, Peyrollier K, Hundal HS. Insulin promotes the cell surface recruitment of the SAT2/ATA2 system $\mathrm{A}$ amino acid transporter from an endosomal compartment in skeletal muscle cells. J Biol Chem. 2002;277(16):13628-13634

102. Ardail D, Gasnier F, Lerme F, Simonot C, Louisot P, Gateau-Roesch O. Involvement of mitochondrial contact sites in the subcellular compartmentalization of phospholipid biosynthetic enzymes. J Biol Chem. 1993;268(34):25985-25992.

103. Bionda C, Portoukalian J, Schmitt D, RodriguezLafrasse C, Ardail D. Subcellular compartmentalization of ceramide metabolism: MAM (mitochondria-associated membrane) and/or mitochondria? Biochem J. 2004;382(pt 2):527-533

104.Shimeno H, Soeda S, Sakamoto M, Kouchi T, Kowakame T, Kihara T. Partial purification and characterization of sphingosine $\mathrm{N}$-acyltransferase (ceramide synthase) from bovine liver mitochondrion-rich fraction. Lipids. 1998;33(6):601-605.

105.Siskind LJ. Mitochondrial ceramide and the induction of apoptosis. J Bioenerg Biomembr. 2005;37(3):143-153.

106. Di Paola M, Cocco T, Lorusso M. Ceramide interaction with the respiratory chain of heart mitochondria. Biochemistry. 2000;39(22):6660-6668.

107.Gudz TI, Tserng KY, Hoppel CL. Direct inhibition of mitochondrial respiratory chain complex III by cell-permeable ceramide. J Biol Chem. 1997;272(39):24154-24158.

108.Garcia-Ruiz C, Colell A, Mari M, Morales A, Fernandez-Checa JC. Direct effect of ceramide on the mitochondrial electron transport chain leads to generation of reactive oxygen species. Role of mitochondrial glutathione. J Biol Chem. 1997; 272(17):11369-11377.

109. Houstis N, Rosen ED, Lander ES. Reactive oxygen species have a causal role in multiple forms of insulin resistance. Nature. 2006;440(7086):944-948.

110. Masharani UB, et al. Insulin resistance in nonobese subjects is associated with activation of the JNK pathway and impaired insulin signaling in skeletal muscle. PLoS One. 2011;6(5):e19878.

111.Yu J, et al. JNK3 signaling pathway activates ceramide synthase leading to mitochondrial dysfunction. J Biol Chem. 2007;282(35):25940-25949.

112.Aerts JM, et al. Pharmacological inhibition of glucosylceramide synthase enhances insulin sensitivity. Diabetes. 2007;56(5):1341-1349.

113.Bijl N, et al. Modulation of glycosphingolipid metabolism significantly improves hepatic insulin sensitivity and reverses hepatic steatosis in mice. Hepatology. 2009;50(5):1431-1441.

114. Bietrix F, et al. Inhibition of glycosphingolipid synthesis induces a profound reduction of plasma cholesterol and inhibits atherosclerosis development in $\mathrm{APOE}^{*} 3$ Leiden and low-density lipoprotein receptor $/-$ mice. Arterioscler Thromb Vasc Biol. 2010; 30(5):931-937.

115.Zhao H, et al. Inhibiting glycosphingolipid synthesis ameliorates hepatic steatosis in obese mice. Hepatology. 2009;50(1):85-93.

116. Glaros EN, Kim WS, Rye KA, Shayman JA, Garner B. Reduction of plasma glycosphingolipid levels has no impact on atherosclerosis in apolipoprotein E-null mice. J Lipid Res. 2008;49(8):1677-1681.

117. Tagami S, et al. Ganglioside GM3 participates in the pathological conditions of insulin resistance. J Biol Chem. 2002;277(5):3085-3092.

118. Langeveld M, de Fost M, Aerts JM, Sauerwein HP, Hollak CE. Overweight, insulin resistance and type 
II diabetes in type I Gaucher disease patients in relation to enzyme replacement therapy. Blood Cells Mol Dis. 2008;40(3):428-432.

119.Ghauharali-van der Vlugt K, et al. Prominent increase in plasma ganglioside GM3 is associated with clinical manifestations of type I Gaucher disease. Clin Chim Acta. 2008;389(1-2):109-113.

120.Mizukami H, et al. Systemic inflammation in glucocerebrosidase-deficient mice with minimal glucosylceramide storage. J Clin Invest. 2002; 109(9):1215-1221.

121. Langeveld M, et al. Type I Gaucher disease, a glycosphingolipid storage disorder, is associated with insulin resistance. J Clin Endocrinol Metab. 2008; 93(3):845-851.

122. Langeveld $M$, et al. Very low serum adiponectin levels in patients with type 1 Gaucher disease without overt hyperglycemia. Metabolism. 2007;56(3):314-319.

123. Kohyama-Koganeya A, Nabetani T, Miura M, Hirabayashi Y. Glucosylceramide synthase (GlcT-1) in the fat body controls energy metabolism in Dro- sophila. J Lipid Res. 2011;52(7):1392-1399.

124.El-Assaad W, et al. Saturated fatty acids synergize with elevated glucose to cause pancreatic beta-cell death. Endocrinology. 2003;144(9):4154-4163.

125. Sjoholm A. Ceramide inhibits pancreatic beta-cell insulin production and mitogenesis and mimics the actions of interleukin-1 beta. FEBS Lett. 1995;367(3):283-286.

126.Kelpe CL, Moore PC, Parazzoli SD, Wicksteed B, Rhodes CJ, Poitout V. Palmitate inhibition of insulin gene expression is mediated at the transcriptional level via ceramide synthesis. J Biol Chem. 2003;278(32):30015-30021.

127.Guo J, Qian Y, Xi X, Hu X, Zhu J, Han X. Blockage of ceramide metabolism exacerbates palmitate inhibition of pro-insulin gene expression in pancreatic beta-cells. Mol Cell Biochem. 2010; 338(1-2):283-290.

128.Shimabukuro M, Higa M, Zhou YT, Wang MY, Newgard CB, Unger RH. Lipoapoptosis in betacells of obese prediabetic $\mathrm{fa} / \mathrm{fa}$ rats. Role of serine palmitoyltransferase overexpression. J Biol Chem. 1998;273(49):32487-32490.

129. Unger RH. Lipotoxic diseases. Annu Rev Med. 2002; 53:319-336.

130. Kolak M, et al. Adipose tissue inflammation and increased ceramide content characterize subjects with high liver fat content independent of obesity. Diabetes. 2007;56(8):1960-1968.

131. Adams JM 2nd, et al. Ceramide content is increased in skeletal muscle from obese insulin-resistant humans. Diabetes. 2004;53(1):25-31.

132. Straczkowski $M$, et al. Increased skeletal muscle ceramide level in men at risk of developing type 2 diabetes. Diabetologia. 2007;50(11):2366-2373.

133. Coen PM, et al. Insulin resistance is associated with higher intramyocellular triglycerides in type I but not type II myocytes concomitant with higher ceramide content. Diabetes. 2010;59(1):80-88.

134. Skovbro M, et al. Human skeletal muscle ceramide content is not a major factor in muscle insulin sensitivity. Diabetologia. 2008;51(7):1253-1260. 